\title{
EFEITO DA FORÇA DA MUSCULATURA RESPIRATÓRIA PRÉ-OPERATÓRIA NO RESULTADO DO TRANSPLANTE DE FÍGADO
}

\author{
Effect of preoperative respiratory muscle strength on he liver transplant outcome
}

\author{
Carla da Silva Machado, Paulo Celso Bosco Massarollo, Eliane Maria de Carvalho, Maria Rita Montenegro Isern, Poliana de \\ Andrade Lima, Sérgio Mies, Aldo Junqueira Rodrigues Jr.
}

\begin{abstract}
RESUMO|
Objetivo: Avaliar o efeito das medidas pré-operatórias das pressões inspiratória máxima (PImáx) e expiratória máxima (PEmáx) no resultado do transplante de fígado (Tx). Métodos: Foram estudados retrospectivamente 228 pacientes submetidos a primeiro Tx eletivo. Os pacientes foram classificados conforme a ocorrência de valores absolutos de pressão respiratória menores ou iguais a $50 \mathrm{~cm} \mathrm{H}_{2} \mathrm{O}$. As variáveis estudadas foram: tempo de ventilação mecânica pós-operatória, necessidade de re-intubação orotraqueal ou de ventilação mecânica não-invasiva, tempo de internação e sobrevida. Resultado: Os resultados mostraram que os valores observados de PImáx e PEmáx estavam abaixo de $50 \mathrm{~cm} \mathrm{H} 2 \mathrm{O}$ em 19,7\% (45/228) e 14,5\% (33/228) dos pacientes, respectivamente. A freqüência de óbito até seis meses após o transplante foi de 26/183 (14,2\%) nos pacientes com PImáx $>50 \mathrm{~cm} \mathrm{H}_{2} \mathrm{O}$ e de $15 / 45(33,3 \%)$ nos pacientes com PImáx mais baixa ( $p=0,003$ ). A sobrevida de 1,3 e 5 anos foi $84 \%, 77 \%$ e $71 \%$ no grupo com PImáx $>50$ cm $\mathrm{H}_{2} \mathrm{O}$ e $57 \%$, $50 \%$ e $50 \%$ no grupo com PImáx mais baixa $(\mathrm{p}=0,0024)$. Em relação à PEmáx, essas probabilidades foram $80 \%, 74 \%$ e $69 \%$ no grupo com valores maiores que 50 $\mathrm{cm} \mathrm{H}_{2} \mathrm{O}$ e $66 \%, 59 \%$ e $51 \%$ nos pacientes com força expiratória menor ( $\left.\mathrm{p}=0,1039\right)$. Não houve diferença estatisticamente significante em relação às demais variáveis analisadas. Conclusão: Pacientes com PImáx baixa apresentam maior mortalidade após o Tx. Entretanto, não foram encontrados efeitos estatisticamente significantes da medida pré-operatória da força da musculatura respiratória nas variáveis de resposta mais diretamente relacionadas com alterações respiratórias.
\end{abstract}

Descritores: Músculos Respiratórios, Debilidade Muscular, Desmame Do Respirador, Transplante de Figado

Instituição:

LIM02 - Departamento de Cirurgia da Faculdade de Medicina da Universidade de São Paulo

Endereço para correspondência:

Carla da Silva Machado

Rua Dr. Jairo de Paula Ferreira, 281 - São José do Rio Preto / SP - CEP: 15091-540 Brasil

Tel.: (17) 32263803 / Fax: (11) 50821064

E-mail: crlmachado@terra.com.br

Recebido em: 10.10 .2007

Aceito em: 10.07.2008

\section{INTRODUÇÃO}

Os músculos desempenham suas funções por meio da produção de movimento ou força. No sistema respiratório, o efeito do encurtamento muscular pode ser avaliado por alterações de volume ou pelo deslocamento da caixa torácica. Já a força é estimada habitualmente por meio das pressões estáticas e dinâmicas envolvidas na mecânica respiratória. Essa prática deve levar em consideração que a eficiência da conversão de força em pressão também depende das características mecânicas das paredes torácica e abdominal, com as quais a musculatura respiratória interage. Assim, as pressões respiratórias devem ser entendidas como um índice de desempenho muscular ao invés de uma medida direta de contratilidade. $^{1}$

A pressão inspiratória máxima (PImáx) é a maior pressão subatmosférica que pode ser gerada durante uma inspiração contra uma via aérea ocluída (manobra de Mueller). ${ }^{2}$ A pressão expiratória máxima (PEmáx) corresponde à pressão desenvolvida durante uma expiração com esforço máximo contra uma via aérea ocluída (manobra de Valsalva). ${ }^{2}$ A medida dessas pressões estáticas é utilizada classicamente para avaliar a força da musculatura respiratória. ${ }^{3}$

Alterações mecânicas da mobilidade da caixa torácica e do diafragma são comuns em cirróticos, secundárias a fatores como ascite e fraqueza muscular. ${ }^{4,5}$ Em pacientes com doença hepática avançada nota-se diminuição da PImáx e da PEmáx em 56\% e $86 \%$ dos casos, respectivamente. ${ }^{6}$ Nos casos com essas alterações, a mortalidade na lista de espera para transplante de fígado (Tx) é 
maior. ${ }^{7}$ Nos pacientes submetidos ao Tx, a diminuição da força dos músculos respiratórios é agravada no pós-operatório imediato. ${ }^{8}$

Em pacientes submetidos à cirurgia de revascularização coronariana, a fraqueza da musculatura respiratória no préoperatório está relacionada com maior dificuldade de desmame e maior incidência de complicações pulmonares. ${ }^{9}$ A eventual ocorrência de efeito semelhante no Tx pode determinar piora do resultado, pois o prolongamento em suporte ventilatório por mais de 24 horas no pós-operatório está associado a aumento de mortalidade, especialmente nos casos que requerem reintubação. ${ }^{10}$ Entretanto, para nosso conhecimento, a importância da medida pré-operatória da força da musculatura respiratória na evolução pósoperatória de pacientes submetidos a Tx ainda não foi investigada. Caso essas variáveis realmente permitam identificar pacientes com maior risco de complicações pulmonares, poderiam ser indicadas medidas de prevenção incluindo desde assistência fisioterápica mais intensa nos períodos pré e pós-operatórios até a escolha de um método operatório mais seguro. ${ }^{11,12}$

O objetivo do presente estudo é avaliar o efeito das medidas préoperatórias de força de musculatura respiratória no resultado do Tx.

\section{MÉTODO}

Foram estudados retrospectivamente todos os $410 \mathrm{Tx}$ realizados pela Unidade de Fígado no Hospital das Clínicas da Faculdade de Medicina da Universidade de São Paulo (HCFMUSP) no período de 28 de dezembro de 1994 a 30 de julho de 2001. Deste grupo, foram excluídos todos os casos nos quais os enxertos foram provenientes de doadores vivos. Esses compreendem $17 \mathrm{Tx}$ intervivos e 18 realizados pela técnica dominó. ${ }^{13,14}$ Também foram excluídos 24 pacientes transplantados por insuficiência hepática aguda grave, 31 retransplantes e 92 casos com dados incompletos. Dessa forma, foram incluídos no estudo 228 pacientes de ambos os sexos sem limite de idade submetidos a primeiro Tx eletivo, com enxerto proveniente de doador cadáver. As etapas de seleção de pacientes para constituição da casuística são mostradas na Figura 1.

Foram revistos os prontuários dos pacientes e anotados os resultados da avaliação das pressões respiratórias estáticas máximas que, segundo a rotina do serviço, eram medidas após a internação para realização do Tx e antes do encaminhamento para o centro cirúrgico. As medidas de PImáx e Pemáx foram obtidas a partir do volume residual (VR) e da capacidade pulmonar total (CPT), respectivamente, utilizando-se um manovacuômetro (Imebraz ${ }^{\circledR}$, modelo MV 150, São Paulo, Brasil) com variação de -150 a $150 \mathrm{cmH}_{2} \mathrm{O}$ adaptado a um bocal com orifício de $1 \mathrm{~mm}$, a fim de prevenir o fechamento da glote e dissipar pressões geradas pela musculatura da face e da orofaringe. ${ }^{3}$ As medidas foram feitas com os pacientes sentados e utilizando clipe nasal. O esforço inspiratório e expiratório foi sustentado por, no mínimo, um segundo. Foram realizadas três medidas consecutivas, sendo registrada a de maior valor. Entretanto, quando a última medida foi maior, o procedimento foi repetido mais uma vez.

Foram anotados também o tempo de ventilação mecânica (definido como o intervalo desde a admissão na UTI, no pós-operatório, até a extubação), a necessidade de retorno ao suporte ventilatório por meio de re-intubação orotraqueal ou de ventilação mecânica não-invasiva e o tempo de internação. Foi verificada a situação dos pacientes em 31 de janeiro de 2002. Em todos os casos foram registradas as datas de realização do transplante e de alta e, nos pacientes que faleceram, a data do óbito.
Figura 1. Diagrama de fluxo mostrando as etapas de seleção de pacientes para constituição da casuística.

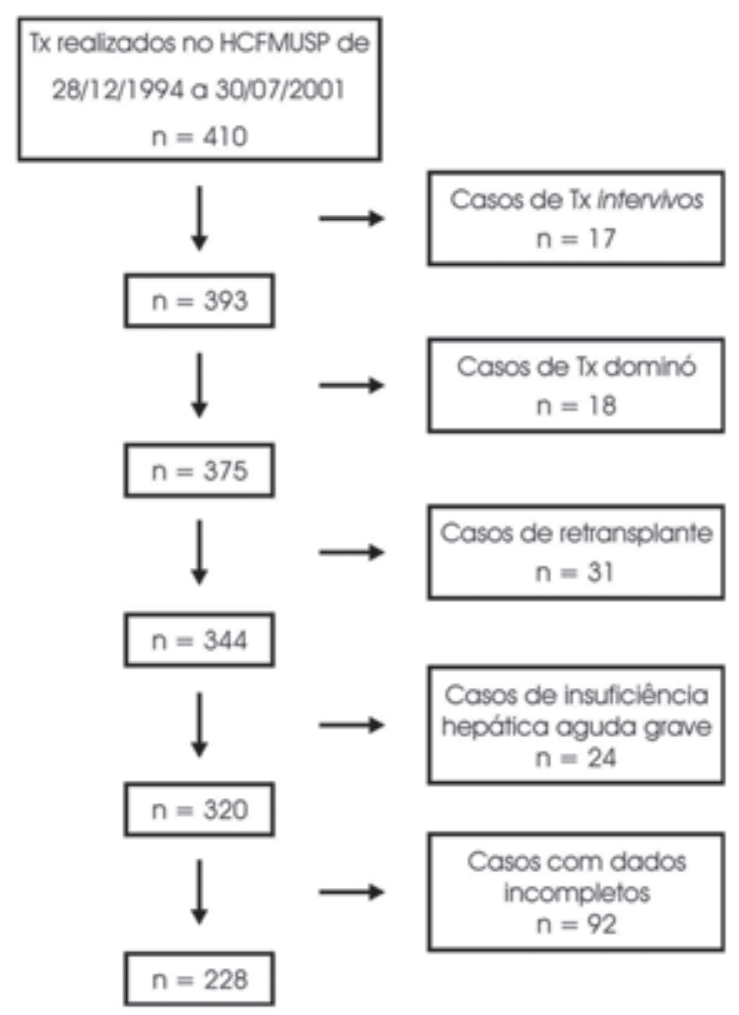

As medidas da Pimáx e da Pemáx foram expressas de dois modos: 1) em valores absolutos; 2) como percentual (\% pred) calculado por meio da razão entre as pressões respiratórias observadas e as preditas utilizando equações de regressão linear publicadas que relacionam as pressões respiratórias máximas com a idade e sexo em indivíduos brasileiros saudáveis. ${ }^{2}$

Os valores observados de Pimáx e PEmáx foram classificados entre normais ou baixos em cada paciente, quando esses valores eram maiores ou menores, respectivamente, do que o resultado da subtração do produto $1,645^{*} \mathrm{RSE}$ (erro padrão residual da estimativa) dos valores preditos pelas equações de regressão. ${ }^{2}$ Pacientes com valores absolutos de Pimáx ou Pemáx menores ou iguais a $50 \mathrm{~cm}$ $\mathrm{H}_{2} \mathrm{O}$ foram classificados como portadores de "fraqueza inspiratória grave" ou "fraqueza expiratória grave", respectivamente.

Adicionalmente, os pacientes foram divididos em grupos conforme a ocorrência ou não de tempo de ventilação mecânica superior a 24 horas, re-intubação orotraqueal, ventilação mecânica não invasiva, insuficiência respiratória (definida como necessidade de ventilação mecânica por mais de 24 horas ou de re-intubação-orotraqueal) e óbito até seis meses após o transplante.

As pressões respiratórias máximas observadas e esperadas foram comparadas por meio do teste t-pareado. A correlação entre as pressões respiratórias máximas e as variáveis quantitativas (tempo de ventilação mecânica e tempo de internação hospitalar) foi analisada por meio do coeficiente de correlação de Spearman $\left(\mathrm{r}_{\mathrm{sp}}\right)$. O efeito dos valores de PImáx e PEmáx nas variáveis qualitativas (tempo de ventilação mecânica superior a 24 horas, re-intubação orotraqueal, ventilação mecânica não invasiva, insuficiência respiratória e 
mortalidade nos primeiros seis meses após transplante) foi estudado por meio de análise de regressão logística. ${ }^{15}$ Os grupos definidos pelos valores de Pımáx e PEmáx (pressão respiratória normal vs. pressão respiratória baixa; com fraqueza respiratória grave vs. sem fraqueza respiratória grave) foram comparados por meio do teste de Mann-Whitney ou do teste qui-quadrado de Pearson, no caso de variáveis quantitativas ou qualitativas, respectivamente. A análise do comportamento temporal da sobrevida de pacientes ao longo do período de acompanhamento foi realizada por meio de construção de curvas atuariais de Kaplan-Meier. ${ }^{16}$ A sobrevida atuarial dos pacientes foi comparada utilizando-se o teste de Cox-Mantel (log-rank) ${ }^{17}$ e o modelo de regressão por tábua de sobrevivência de Cox. ${ }^{18}$

Os 228 pacientes incluídos foram utilizados na análise das variáveis de resposta qualitativas. Em relação às variáveis de resposta quantitativas nas comparações referentes ao "tempo de ventilação mecânica", foram excluídos da amostra quatro pacientes que faleceram no período intra-operatório. Da mesma forma, foram excluídos da análise da variável "tempo de internação" 18 pacientes que faleceram até 11 dias após o transplante. Esse limite foi definido por coincidir com o tempo de internação mínimo exigido pelo Ministério da Saúde para pacientes submetidos a Tx por meio do Sistema Único de Saúde. Em nossa casuística, a população dependente dessa cobertura corresponde a cerca de $90 \%$ dos pacientes.

As análises estatísticas foram realizadas utilizando o programa SPSS for Windows versão 9.0 (Statistical Package for the Social Sciences Inc., Chicago, IL, EUA). Foi utilizado nível de significância de $5 \%$. Os valores são expressos em média \pm desvio padrão.

\section{RESULTADO}

A idade média dos 228 pacientes estudados foi 46,2 \pm 13,7 anos (limites nove a 75 anos). Havia na amostra 136 pacientes do sexo masculino (59,7\%). A principal etiologia da doença hepática era hepatite viral em 98 casos $(43,0 \%)$, doenças colestáticas em 33 (14,5\%), álcool em 30 (13,2\%), hepatite auto-imune em 16 (7,0\%) e outras causas nos demais 51 pacientes $(22,4 \%)$. Em relação à gravidade da doença hepática, 38 pacientes $(16,7 \%)$ foram classificados como Child-Pugh A, 125 (54,8\%) Child-Pugh B e 65 (28,5\%) Child-Pugh C.

Os valores de PImáx observados (79,5 \$33,6 cm H2O) e preditos $(105,6$ $\left.\pm 16,0 \mathrm{~cm} \mathrm{H}_{2} \mathrm{O}\right)$ apresentam diferença estatisticamente significante $(95 \% \mathrm{CI}=21,9$ a 30,2; $<<0,001)$. Também é estatisticamente significante a diferença entre os valores observados $\left(86,4 \pm 31,8 \mathrm{~cm} \mathrm{H}_{2} \mathrm{O}\right)$ e preditos $\left(111,1 \pm 20,2 \mathrm{~cm} \mathrm{H}_{2} \mathrm{O}\right)$ da Pemáx $(95 \% \mathrm{CI}=21,2$ a 28,5; $\mathrm{p}<0,001)$.

Os valores médios de Pimáx (\% pred) e Pemáx (\% pred) foram $75,3 \pm 30,1 \%$ e $77,6 \pm 25,9 \%$, respectivamente. A distribuição dos valores de Pimáx (\% pred) e Pemáx (\% pred) é mostrada na figura 2. Os valores observados de Pimáx e Pemáx estavam abaixo do limite normal em $61 \%(139 / 228)$ e $55 \%(126 / 228)$ dos pacientes, respectivamente. Os valores observados de Pimáx e Pemáx foram menores que $50 \mathrm{~cm} \mathrm{H}_{2} \mathrm{O}$ em 19,7\% (45/228) e 14,5\% (33/228) dos pacientes, respectivamente.

Não houve correlação significante entre a PImáx (\% pred) e o tempo de ventilação mecânica $\left(r_{\mathrm{sp}}=0,0397 ; \mathrm{p}=0,554\right)$ e o tempo de internação hospitalar $\left(\mathrm{r}_{\mathrm{sp}}=-0,0490 ; \mathrm{p}=0,480\right)$. Também não houve correlação significante entre a PEmáx (\% pred) e o tempo de ventilação mecânica $\left(\mathrm{r}_{\mathrm{sp}}=-0,0123 ; \mathrm{p}=0,854\right)$ e o tempo de internação hospitalar $\left(\mathrm{r}_{\mathrm{sp}}=-0,0432 ; \mathrm{p}=0,534\right)$.
Figura 2. Gráfico box-plot mostrando a distribuição dos valores de PImáx (\% pred) e PEmáx (\% pred).

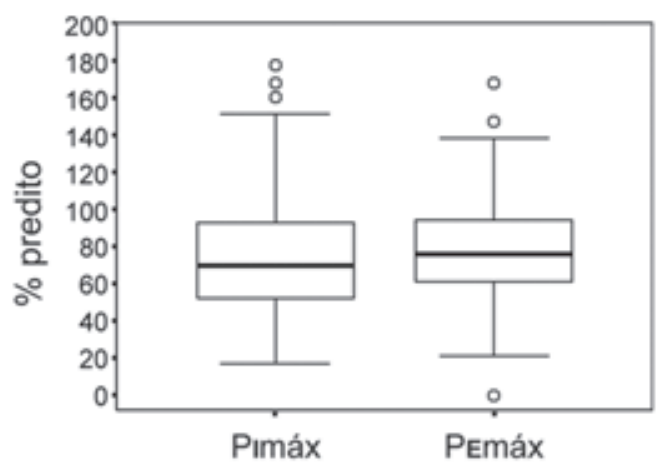

$\mathrm{Na}$ análise de regressão logística, não houve interação significante entre os valores da PImáx (\% pred) e o tempo de ventilação mecânica superior a 24 horas (odds ratio $=1,000$; IC95\% $=0,992$ a 1,009; $\mathrm{p}=0,946)$, necessidade de re-intubação orotraqueal (odds ratio = 1,000; IC95\% = 0,991 a 1,009; $\mathrm{p}=0,995)$, necessidade de ventilação mecânica não invasiva (odds ratio = 1,004; IC95\%=0,996 a 1,013; $\mathrm{p}=0,432$ ), insuficiência respiratória (odds ratio $=1,002 ;$ IC95\% $=$ $0,995$ a 1,009; $\mathrm{p}=0,670)$, e mortalidade nos primeiros 6 meses após o transplante (odds ratio $=0,990 ;$ IC95\% $=0,980$ a 1,001; $\mathrm{p}=0,121$ ). Também não houve interação significante entre os valores de Pemáx (\% pred) e o tempo de ventilação mecânica superior a 24 horas (odds ratio $=0,994 ;$ IC95\% $=0,984$ a 1,004; $\mathrm{p}=0,330$ ), necessidade de re-intubação orotraqueal (odds ratio $=0,995 ;$ IC95\% $=0,985$ a $1,005 ; \mathrm{p}=0,431)$, necessidade de ventilação mecânica não invasiva (odds ratio =0,999; IC95\% =0,989 a 1,009; $\mathrm{p}=0,831$ ), insuficiência respiratória (odds ratio $=0,997 ;$ IC95\% $=0,989$ a 1,$006 ; p=0,621$ ) e mortalidade nos primeiros 6 meses após o transplante (odds ratio $=0,989 ;$ IC95\% $=0,978$ a 1,$001 ; p=0,127$ ).

A freqüência de óbito até seis meses após o transplante foi de 10/89 (11,2\%) em pacientes com PImáx normal e de 31/139 (22,3\%) em pacientes com Pimáx baixa $(\mathrm{p}=0,034)$. Os dois grupos não apresentaram diferença estatisticamente significante em relação à duração mediana do tempo de ventilação mecânica (850 vs.810 minutos; $p=0,291$ ) e do tempo de internação (18 vs. 17 dias; $p=0,843)$, bem como em relação à freqüência de ventilação mecânica por mais de 24 horas $(23 / 89$ vs. 30/139; $\mathrm{p}=0,458)$, re-intubação orotraqueal (19/89 vs. 35/139; $p=0,507)$, ventilação mecânica não invasiva $(22 / 89$ vs. $30 / 139 ; \mathrm{p}=0,582)$ e insuficiência respiratória (35/89 vs. 51/139; $\mathrm{p}=0,689)$. Os grupos de pacientes com Pemáx normal ou baixa não apresentaram diferença estatisticamente significante em relação à duração mediana do tempo de ventilação mecânica (820 vs.840 minutos; $p=0,776)$ e do tempo de internação (19 vs.16,5 dias; $\mathrm{p}=0,851)$, bem como em relação à freqüência de ventilação mecânica por mais de 24 horas $(24 / 102$ vs.29/126; $\mathrm{p}=0,927)$, reintubação orotraqueal $(24 / 102$ vs.30/126; $\mathrm{p}=0,961)$, ventilação mecânica não-invasiva $(22 / 102$ vs.30/126; $\mathrm{p}=0,688)$, insuficiência respiratória (40/89 vs.46/139; $\mathrm{p}=0,675)$ e mortalidade nos primeiros 6 meses após o transplante $(16 / 102$ vs.25/126; $\mathrm{p}=0,417)$.

A freqüência de óbito até seis meses após o transplante foi de 15/45 (33,3\%) nos pacientes com Pimáx $\leq 50 \mathrm{~cm} \mathrm{H}_{2} \mathrm{O}$ e de 26/183 $(14,2 \%)$ nos pacientes com Pimáx $>50 \mathrm{~cm} \mathrm{H}_{2} \mathrm{O}(\mathrm{p}=0,003)$. Os dois grupos não apresentaram diferença estatisticamente significante 
em relação à duração mediana do tempo de ventilação mecânica (830,0 vs. 805,0 minutos; $p=0,729)$ e do tempo de internação $(17,0$ vs.17,0 dias; $\mathrm{p}=0,505)$, bem como em relação à freqüência de ventilação mecânica por mais de 24 horas ( $9 / 45$ vs. 44/183; $p=0,565)$, re-intubação orotraqueal (14/45 vs. 44/183; p=0,191), ventilação mecânica não-invasiva (10/45 vs. 42/183; p=0,917) e insuficiência respiratória $(18 / 45$ vs. 68/183; $\mathrm{p}=0,725)$. Os grupos de pacientes com Pemáx $\leq 50 \mathrm{~cm} \mathrm{H}_{2} \mathrm{O}$ e Pemáx $>50 \mathrm{~cm} \mathrm{H}_{2} \mathrm{O}$ não apresentaram diferença estatisticamente significante em relação à duração mediana do tempo de ventilação mecânica $(835,0$ vs.827,5 minutos; $\mathrm{p}=0,879)$ e do tempo de internação $(18,5$ vs.17,0 dias; $\mathrm{p}=0,212)$, bem como em relação à freqüência de ventilação mecânica por mais de 24 horas $(8 / 33$ vs.45/195; p=0,883), re-intubação orotraqueal (11/33 vs.43/195; $p=0,159)$, ventilação mecânica não-invasiva $(10 / 33$ vs.42/195; $\mathrm{p}=0,267)$, insuficiência respiratória (14/33 vs.72/195; $\mathrm{p}=0,547)$ e mortalidade nos primeiros 6 meses após o transplante (8/33 vs.33/195; $\mathrm{p}=0,311)$.

As curvas de Kaplan-Meier com a sobrevida atuarial dos pacientes com PImáx normal ou baixa e com Pemáx normal ou baixa são mostradas nas Figuras 3 e 4 . A sobrevida de 1, 3 e 5 anos foi $86 \%, 79 \%$ e $66 \%$ no grupo com Pimáx normal e $73 \%, 67 \%$ e $66 \%$ no grupo com PImáx baixa $(\mathrm{p}=0,2419)$. Em relação à PEmáx, essas probabilidades foram $82 \%, 77 \%$ e $72 \%$ no grupo com valores normais e $75 \%, 68 \%$ e $62 \%$ nos pacientes com força expiratória diminuída $(p=0,1114)$. A análise de regressão proporcional de Cox não indicou maior risco de

Figura 3. Curva de Kaplan-Meier mostrando a sobrevida atuarial após o transplante de fígado nos pacientes com Plmáx normal ou baixa.

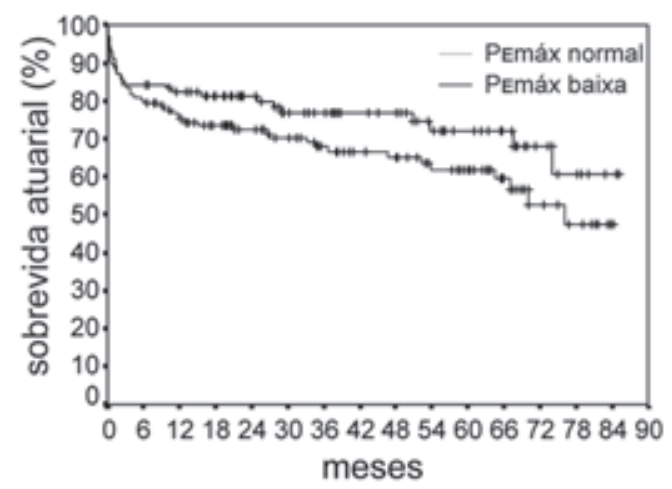

Figura 4. Curva de Kaplan-Meier mostrando a sobrevida atuarial após o transplante de fígado nos pacientes com PEmáx normal ou baixa.

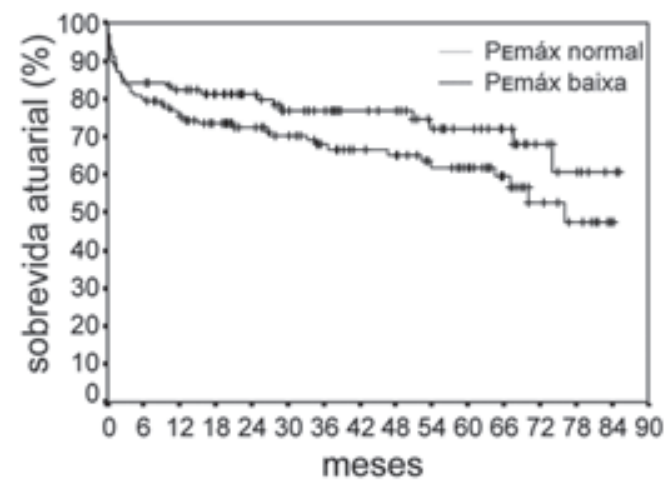

mortalidade nos pacientes com PImáx baixa (risco relativo $=1,3377$; IC95\% $=0,8193$ a 2,1841; $\mathrm{p}=0,2448$ ) ou com Pemáx baixa (risco relativo $=1,4729 ; \mathrm{IC} 95 \%=0,9105$ a 2,2328; $\mathrm{p}=0,1146$ ).

As curvas de Kaplan-Meier com a sobrevida atuarial dos pacientes com Pimáx $\leq 50 \mathrm{~cm} \mathrm{H}_{2} \mathrm{O}$ e com Pimáx $>50 \mathrm{~cm} \mathrm{H}_{2} \mathrm{O}$ são mostradas na Figura 5. A sobrevida de 1, 3 e 5 anos foi $84 \%$, $77 \%$ e $71 \%$ no grupo com Pimáx $>50 \mathrm{~cm} \mathrm{H}_{2} \mathrm{O}$ e $57 \%, 50 \%$ e $50 \%$ no grupo com Pimáx $\leq 50 \mathrm{~cm} \mathrm{H}_{2} \mathrm{O}(\mathrm{p}=0,0024)$. As curvas de Kaplan-Meier com a sobrevida atuarial dos pacientes com Pemáx $\leq 50 \mathrm{~cm} \mathrm{H}_{2} \mathrm{O}$ e com Pemáx $>50 \mathrm{~cm} \mathrm{H} \mathrm{H}_{2} \mathrm{O}$ são mostradas na Figura 6. A sobrevida de 1, 3 e 5 anos foi $80 \%$, 74\% e $69 \%$ no grupo com Pemáx $>50 \mathrm{~cm} \mathrm{H}_{2} \mathrm{O}$ e $66 \%$, 59\% e 51\% no grupo com Pemáx $\leq 50 \mathrm{~cm} \mathrm{H2O} \mathrm{(p=0,1039).} \mathrm{A} \mathrm{análise} \mathrm{de} \mathrm{regressão}$ proporcional de Cox indicou risco significantemente maior de mortalidade nos pacientes com PImáx $\leq 50 \mathrm{~cm} \mathrm{H}_{2} \mathrm{O}$ (risco relativo $=2,1696$; IC95\% = 1,2987 a 3,6347; $\mathrm{p}=0,0031$ ), mas não nos casos com Pemáx $\leq 50 \mathrm{~cm} \mathrm{H}_{2} \mathrm{O}$ (risco relativo = 1,6172; IC95\% $=0,8995$ a 2,$9075 ; \mathrm{p}=0,1083)$.

\section{DISCUSSÃO}

O resultado do presente estudo confirma achados de publicações anteriores, que relatam diminuição da força dos músculos respiratórios em pacientes em estágio avançado de doença hepática. ${ }^{4-6,19}$ Esse fenômeno pode ser explicado por algumas alterações freqüentes nessa população, como ascite e desnutrição.

Figura 5. Curva de Kaplan-Meier mostrando a sobrevida atuarial após o transplante de fígado nos pacientes com PImáx $\leq 50 \mathrm{~cm} \mathrm{H_{2 }} \mathrm{O}$ ou PImáx $>50 \mathrm{~cm} \mathrm{H} \mathrm{H}_{2} \mathrm{O}$

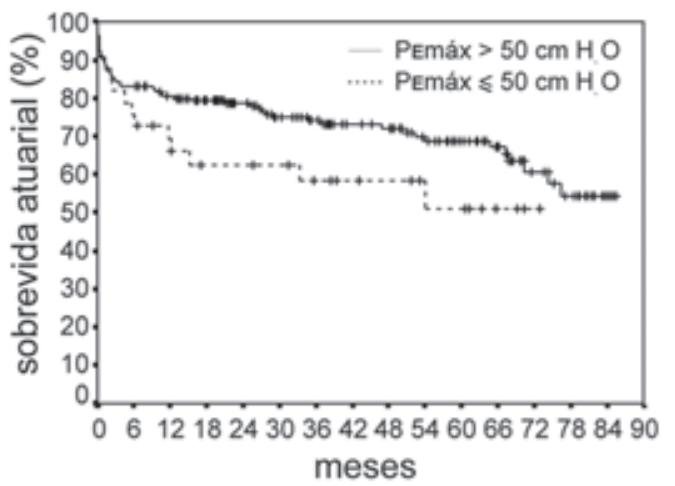

Figura 6. Curva de Kaplan-Meier mostrando a sobrevida atuarial após o transplante de fígado nos pacientes com PEMáx $\leq 50 \mathrm{~cm} \mathrm{H_{2 } O}$ ou PEmáx $>50 \mathrm{~cm} \mathrm{H} \mathrm{H}_{2} \mathrm{O}$.

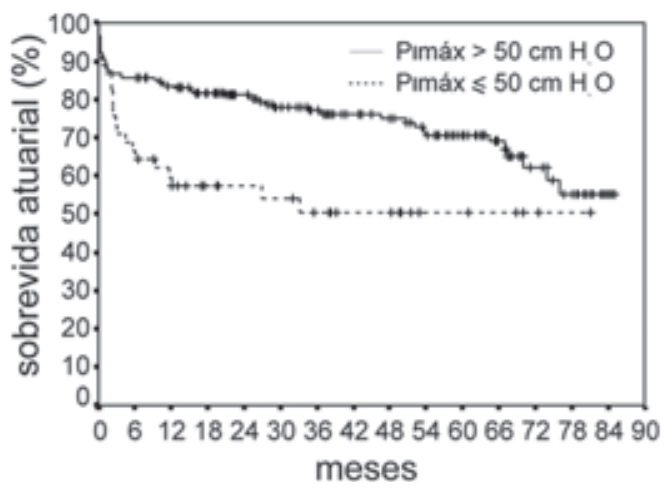


A presença de ascite tensa determina aumento da pressão intraabdominal que é transmitida para o tórax, causando aumento da pressão pleural, elevação do diafragma e diminuição da mobilidade das paredes do tórax. ${ }^{4}$ Os movimentos mais rígidos da caixa torácica requerem aumento do trabalho respiratório, resultando em um padrão ventilatório restritivo. ${ }^{4,5}$ Apesar dessas alterações na mecânica respiratória poderem justificar a diminuição da Pımáx e da Pemáx, essas pressões não retornam à normalidade após a realização de paracentese esvaziadora, ${ }^{4,5}$ sugerindo que outros fatores devam estar envolvidos. Em pacientes adultos inscritos na lista de espera para transplante de fígado, a prevalência de desnutrição é superior a 50\% ${ }^{20}$ Arora e Rochester observaram que tanto a Pimáx quanto a Pemáx, são diminuídas em indivíduos desnutridos quando comparados a controles normais. ${ }^{21}$

Em cirurgias abdominais altas, a diminuição da PImáx é um evento previsto, com expectativa de retorno gradual à normalidade em um prazo de 48 horas a uma semana após a cirurgia. ${ }^{22}$ Lima e cols. estudaram as alterações da mecânica respiratória no pós-operatório imediato do transplante de fígado, observando que no $10^{\circ}$ dia pós-operatório a medida de PImáx ainda não havia retornado aos valores aferidos no pré-operatório. ${ }^{8}$ Plank e cols. avaliaram a força muscular de pacientes submetidos a transplante de fígado. ${ }^{23} \mathrm{~A}$ força das mãos não somente aumentou como superou os valores aferidos no pré-operatório em aproximadamente três meses. Em contrapartida, a força dos músculos respiratórios apresentou pouca melhora, sendo significantemente menor do que os níveis normais após 12 meses. $^{23}$

No pós-operatório imediato, a redução da força da musculatura respiratória pode dificultar o processo de desmame da ventilação mecânica. ${ }^{24}$ Pacientes que permanecem em ventilação mecânica por mais de $24 \mathrm{hs}$ ou necessitam de reintubação apresentam menor sobrevida. ${ }^{10} \mathrm{~A}$ disfunção dos músculos respiratórios pode provocar uma redução na capacidade vital, no volume total e na capacidade pulmonar total. ${ }^{22}$ Nos pacientes com diminuição da atuação dos músculos expiratórios, a tosse pode se tornar ineficaz, dificultando a realização da adequada higiene brônquica de maneira independente. Já a diminuição da força dos músculos inspiratórios dificulta a expansão pulmonar favorecendo a formação de pequenas atelectasias, comuns nos pacientes cirúrgicos. A presença de atelectasias pode representar um fator de risco para infecções pulmonares, ${ }^{22}$ situação agravada em pacientes que fazem uso de drogas imunossupressoras para combater a rejeição do enxerto, como é o caso dos receptores de transplante de fígado. $\mathrm{O}$ emprego de corticóides pode também contribuir adicionalmente para a piora da performance dos músculos respiratórios. ${ }^{25}$

No presente estudo, as forças respiratórias foram expressas e analisadas utilizando tanto valores relativos como absolutos. Apesar do achado de maior mortalidade até seis meses após Tx nos casos com Pimáx baixa, as curvas de sobrevida atuarial de pacientes com pressões respiratórias máximas normais ou baixas não apresentaram diferença estatisticamente significante. Já os pacientes com valores absolutos de Pimáx $\leq 50 \mathrm{~cm} \mathrm{H}_{2} \mathrm{O}$ apresentaram sobrevida significantemente menor. Essa discrepância deve ser interpretada considerando as diferenças entre os dois critérios de classificação. Na primeira situação, os pacientes foram classificados em relação a padrões de normalidade estabelecidos para brasileiros saudáveis de mesma idade e sexo. ${ }^{2}$ Embora essa abordagem seja adequada para finalidades epidemiológicas, pode apresentar limitações nas aplicações funcionais da medida da Pımáx e da Pemáx como o estudo pré-operatório da função dos músculos ventilatórios, avaliação do resultado de treinamento muscular respiratório e critérios de desmame da ventilação mecânica. ${ }^{9,24}$ Nessas situações particulares, torna-se relevante definir valores absolutos que permitam uma respiração espontânea adequada ao invés de apenas categorizar os indivíduos conforme a normalidade.

Tome-se, por exemplo, a questão do desmame da ventilação mecânica. O sucesso desse processo depende de valores mínimos da força dos músculos respiratórios, considerados habitualmente a redor de $30 \mathrm{~cm} \mathrm{H}_{2} \mathrm{O} .{ }^{24}$ Valores ainda maiores podem ser necessários, caso estejam presentes desordens de fatores como carga aplicada sobre os músculos e atividade do sistema nervoso central. ${ }^{24}$ Percebese que um indivíduo idoso pode ser classificado como normal para a faixa etária, mas apresentar valores absolutos de força respiratória que determinem dificuldade de desmame da ventilação mecânica. Inversamente, indivíduos jovens, embora com pressões abaixo das esperadas para a idade, podem ter valores absolutos suficientes para garantir a respiração espontânea.

Hourani e cols, ${ }^{6}$ analisando a função pulmonar em pacientes com doença hepática avançada definiram "fraqueza respiratória grave" valores absolutos de força muscular inferiores a $40 \mathrm{~cm} \mathrm{H}_{2} \mathrm{O}$. No presente estudo, utilizou-se limite de $50 \mathrm{~cm} \mathrm{H}_{2} \mathrm{O}$, considerando a diminuição esperada da Pimáx e da Pemáx após o transplante de $32 \%$ e $42 \%$, respectivamente. ${ }^{8}$

Apesar da interação entre Pımáx e mortalidade, não foram encontrados efeitos estatisticamente significantes das pressões respiratórias máximas em relação às variáveis mais diretamente relacionadas com alterações respiratórias, como tempo de ventilação mecânica, incidência de insuficiência respiratória e tempo de internação hospitalar. O resultado negativo pode estar relacionado com interferência de outras alterações clínicas nas variáveis de resposta analisadas.

Embora o tempo de ventilação mecânica e a necessidade de reintubação oro-traqueal tenham sido utilizados para definir insuficiência respiratória, esses eventos não dependem apenas da troca gasosa e da mecânica respiratória. Glanemann observou que de 110 casos de reintubação oro-traqueal no pós-operatório do transplante de fígado, causas pulmonares foram a indicação em 49 casos. ${ }^{26}$ As demais dividiram-se 24 neurológicas, 16 cirúrgicas, 10 cardíacas e 11 por causas diversas. ${ }^{26}$

A ocorrência de complicações neurológicas representadas por alterações do estado mental, convulsões, déficit motor é comum após transplante de fígado. ${ }^{27}$ Essas complicações têm sido atribuídas a fatores como disfunção do enxerto, hemorragias intracranianas, acidente vascular cerebral, infecção e toxidade pelas drogas imunossupressoras. ${ }^{27} \mathrm{~A}$ falência renal é outra complicação freqüente que está relacionada à instabilidade hemodinâmica e maior mortalidade especialmente nos casos que requerem o uso de diálise. ${ }^{28}$ Essas alterações podem determinar dificuldade para o desmame da ventilação mecânica ou necessidade de reintubação.

\section{CONCLUSÃO}

Conclui-se que pacientes com Pımáx baixa apresentam maior mortalidade após o transplante de fígado. Entretanto, não foram encontrados efeitos estatisticamente significantes da medida préoperatória da força da musculatura respiratória nas variáveis de resposta mais diretamente relacionadas com alterações respiratórias. 


\section{ABSTRACT}

Purpose: To assess the effect of the preoperative respiratory muscle strength on the orthotopic liver transplantation (OLT) outcome. Methods: Maximal inspiratory pressure (PImax) and maximal expiratory pressure (PEmax) were assessed in 228 recipients immediately before OLT. Patients were classified according to the occurrence of the absolute values of muscle strength equal or lower than $50 \mathrm{~cm}_{2} \mathrm{O}$. The following response variables were analyzed: duration of postoperative mechanical ventilation, incidence of tracheal reintubation and noninvasive positive pressure ventilation, length of hospital stay, and patient survival. Results: PImax e PEmax were equal or lower than 50 $\mathrm{cm} \mathrm{H}_{2} \mathrm{O}$ in $19.7 \%$ (45/228) and 14.5\% (33/228) of patients, respectively. The patient mortality up to 6 months after OLT was $14.2 \%(26 / 183)$ in the group with PImax $>50 \mathrm{~cm} \mathrm{H}_{2} \mathrm{O}$ and 33.3\% (15/45) in the group with lower values ( $\left.\mathrm{p}=0.003\right)$. The 1-, 3-, and 5-year patient survival was $84 \%, 77 \%$ and $71 \%$ for the group with PImax $>50 \mathrm{~cm} \mathrm{H}_{2} \mathrm{O}$ and $57 \%, 50 \%$ and $50 \%$ for the group with lower values ( $\mathrm{p}=0.0024$ ). As to the PEmax, probabilities were $80 \%, 74 \%$ and $69 \%$ for the group with higher values and $66 \%, 59 \%$ and $51 \%$ for patients with respiratory muscle weakness $(\mathrm{p}=0.1039)$. There was no significant difference regarding others variables analyzed. Conclusion: Patients with low PImax present higher mortality rates after OLT. However, there are no statistically significant effects of the preoperative respiratory muscle strength on the response variables more directly related to the pulmonary outcome.

Keywords: Respiratory Muscles, Muscle Weakness, Ventilator Weaning, Liver Transplantation

\section{REFERÊNCIAS}

1. American Thoracic Society/European Respitatory Society. ATS/ERS Statement on respiratory muscle testing. Am J Respir Crit Med. 2002;166:518-624.

2. Neder JA, Andreoni S, Lerario MC, Nery LE. References values for lung function tests. II. Maximal respiratory pressures and voluntary ventilation. Braz J Med Biol Res. 1999;32:719-27.

3. Black LF, Hyatt RE. Maximal respiratory pressures: normal values and relationship to age and sex. Am Rev Respir Dis. 1969;99:696-702.

4. Duranti R, Laffi G, Misuri G, Gorini M, Foschi M, Iandelli I, et al. Respiratory mechanics in patients with tense cirrhotic ascites. Eur Respir J. 1997;10:1622-30.

5. Ordiales Fernández JJ, Fernández Moya A, Nistal de Paz F, Linares Rodríguez A, Colubi Colubi L, Alvarez Ascensio E, et al. Influencia de la cirrosis hepática com y sin ascitis sobre la mecánica ventilatoria. Rev Esp Enfem Digest .1995;87:853-7.

6. Hourani JM, Bellamy PE, Tashkin DP, Batra P, Simmons MS. Pulmonary dysfunction in advanced liver disease: frequent occurrence of an abnormal diffusing capacity. Am J Med. 1991;90:693-700.

7. Carvalho EM, Lima PA, Machado CS, Massarollo PCB, Isern MRM, Dezan FG, et al. Muscle strength and mortality while on the waiting list for liver transplantation. Liver Transpl. 2000;6:37.

8. Lima PA, Carvalho EM, Isern MRM, Massarollo PCB, Mies S. Mecânica respiratória e oxigenacão no transplante de fígado. J Pneumol. 2002;28 (suppl 2):P39

9. Weiner P, Zeidan F, Zamir D, Pelled Benny, Waizman J, Beckerman M, et al. Prophylatic inspiratory muscle training in patients undergoing coronary artery bypass graft. World J Surg. 1998;22:427-31.

10. Glanemann M, Langrehr JM, Kaisers U, Schenk R, Müller A, Stange B, et al. Postoperative tracheal extubation after orthotopic liver transplantation. Acta Anaesthesiol Scand. 2001;45:333-39.

11. Carvalho EM, Massarollo PCB, Isern MRM, Toledo NS, Kawacama J, Mies S, et al. Pulmonary evolution in conventional liver transplantation with venovenous bypass and the piggyback method. Transplant Proc. 1999;31:3064-6.

12. Isern MRM, Massarollo PCB, Carvalho EM, Baia CE, Kavakama J, Lima PA, et al. Randomized trial comparing pulmonary alterations after conventional with venovenous bypass versus piggyback liver transplantation. Liver Transpl. 2004,10:425-33.

13. Raia S, Nery JR, Mies S. Liver transplantation from live donors. Lancet. 1989; 2:497.

14. Azoulay D, Samuel D, Castaing D, Adam R, Adams D, Said G, et al. Domino liver transplants for metabolic disorders: experience with familial amyloidotic polyneuropathy. J Am Coll Surg. 1999;189:584-93.
15. Christensen R. Log-Linear Models and Logistic Regression. New York: SpringerVerlag 1997.

16. Kaplan EL, Meier P. Nonparametric estimation from incomplete observations. J Am Stat Assoc. 1958;53:457-81.

17. Friedman LM, Furberg CD, DeMets DL. Survival analysis. In.: Friedman LM, Furberg CD, DeMets DL. Fundamentals of clinical trials. New York: Spriger-Verlag. 1998:233-45.

18. Cox DR. Regression models and lifetables. J R Stat Soc, Series B 1972;34:187-202.

19. Filintrop M, Modi MR, Ritter AB, Duran W, Lavietes MH. Respiratory muscle length and strength in patients with chronic abdominal distension. Respiration. 1997;64:66-72.

20. Le Cornu KA, McKiernan JF, Kapadia SA, Neuberger JM. A prospective randomized study of preoperative nutritional supplementation in patients awaiting elective orthotopic liver transplantation. Transplantation. 2000;69:1364-9.

21. Arora NS, Rochester DF. Respiratory muscle strength and maximal voluntary ventilation in undernourished patients. Am Rev Respir Dis. 1982;126:5-8.

22. Siafakas NM, Mitrouska I, Bouros D, Georgopoulos D. Surgery and the respiratory muscles. Thorax. 1999;54:458-65.

23. Plank LD, Metzger DJ, McCall JL, Barclay K, Gane EJ, Streat SJ, et al. Sequential changes in the metabolic response to orthotopic liver transplantation during the first year after surgery. Ann Surg. 2001;22:245-55.

24. Carvalho EM, Lima PA, Isern MRM, Mies S, Massarollo PCB, Raia S. Evolution of predictive weaning indices for mechanical ventilation in liver transplantation. Transplant Proc. 1999;31:3053-4.

25. Weiner P, Azgad Y, Weiner M. Inspiratory muscle training during treatment with corticosteroids in humans. Chest. 1995;107:1041-4.

26. Glanemann M, Kaisers U, Langrehr JM, Schenk R, Stange BJ, Müller AR, et al. Incidence and indications for reintubation during postoperative care following ortothopic liver transplantation. J Clin Anesth. 2001;13:377-82.

27. Ardizzone G, Arrigo A, Schellino MM, Stratta C. Valzan S, Skurzak S, et al. Neurological complications of liver cirrhosis and orthotopic liver transplant. Transplant Proc. 2006;38:789-92.

28. Lima EQ, Zanetta DM, Castro I, Massarollo PCB, Mies S, Machado MM, et al. Risk factors for development of acute renal failure after liver transplantation Ren Fail. 2003;25:553-60. 